\title{
Training to enhance psychiatrist communication with patients with psychosis (TEMPO): cluster randomised controlled trial
}

Rose Mccabe, Paula John, Jemima Dooley, Patrick Healey, Annie Cushing, David Kingdon, Stephen Bremner and Stefan Priebe

\section{Background}

A better therapeutic relationship predicts better outcomes. However, there is no trial-based evidence on how to improve therapeutic relationships in psychosis.

\section{Aims}

To test the effectiveness of communication training for psychiatrists on improving shared understanding and the therapeutic relationship (trial registration: ISRCTN94846422).

\section{Method}

In a cluster randomised controlled trial in the UK, 21 psychiatrists were randomised. Ninety-seven (51\% of those approached) out-patients with schizophrenia/schizoaffective disorder were recruited, and 64 (66\% of the sample recruited at baseline) were followed up after 5 months. The intervention group received four group and one individualised session. The primary outcome, rated blind, was psychiatrist effort in establishing shared understanding (self-repair) secondary outcome was the therapeutic relationship.

\section{Results}

Psychiatrists receiving the intervention used $44 \%$ more self-repair than the control group (adjusted difference in means $6.4,95 \% \mathrm{Cl} 1.46-11.33, P<0.011$, a large effect) adjusting for baseline self-repair. Psychiatrists rated the therapeutic relationship more positively (adjusted difference in means $0.20,95 \% \mathrm{Cl} 0.03-0.37, P=0.022$, a medium effect) as did patients (adjusted difference in means 0.21 , $95 \% \mathrm{Cl}$ 0.01-0.41, $P=0.043$, a medium effect).

\section{Conclusions}

Shared understanding can be successfully targeted in training and improves relationships in treating psychosis.

\section{Declaration of interest}

None.

\section{Copyright and usage}

(c) The Royal College of Psychiatrists 2016.
A better therapeutic relationship in the treatment of psychosis predicts better treatment adherence, less severe symptoms, better social functioning and fewer hospital admissions. ${ }^{1-4} \mathrm{~A}$ meta-analysis found the odds of a patient adhering to treatment to be 2.16 times greater if there is a good doctor-patient relationship. ${ }^{5}$ However, although patients rate the therapeutic relationship as the most important component of psychiatric care ${ }^{6}$ currently there are no trial-based interventions for how psychiatrists can improve the therapeutic relationship.

The psychiatrist-patient therapeutic relationship is negotiated in psychiatrist-patient communication, and psychiatrists consider effective communication skills to be one of the most important characteristics of a good psychiatrist. ${ }^{1,7}$ Hence, improving communication is central to improving the relationship. Using conversation analysis, a method increasingly applied to medicine which analyses what people do rather than what they say they do, ${ }^{8,9}$ previous research identified a lack of shared understanding in psychiatrist-patient communication in the treatment of psychosis. This often centred on exchanges about psychotic symptoms in the context of mental state assessment. Patients repeatedly attempted to discuss the content and emotional consequences of their hallucinations and delusions, whereas psychiatrists tended to avoid engaging with these concerns in an attempt to avoid disagreement. This led to patients asking direct questions about these experiences (e.g. 'Why don't people believe me?', 'Do you believe me?') in an attempt to establish a shared understanding. ${ }^{10}$

One specific index of good communication is 'self-repair', explained in detail elsewhere. ${ }^{11,12}$ This is a conversation analytic term, which reflects attempts to achieve shared understanding. It refers to the 'online' processes of editing or reworking an utterance while it is being produced. Self-repairs are ubiquitous in natural, unscripted dialogue and have proved to be a useful measure of how hard people are working to make their talk understandable and acceptable to the listener in conversation generally and in psychiatric encounters. ${ }^{13,14}$ For example, in the following excerpt, the psychiatrist asks the patient about reducing or stopping his procyclidine, reformulating the utterance as he produces it.

I mean [1] what if we, ah erhhh [2] what would your thoughts be about - [3] what if I said to you well we should look about reducing them or stopping them, what would you think about that?

His first formulation 'what if we' is abandoned and reworked as 'what would your thoughts be about'. This is reworked again as 'what if I said to you well we should look about reducing or stopping them?'. The final formulation presents a hypothetical proposal for the patient to consider, indicating that the subsequent action will take the patient's position into account. In a previous observational study of psychiatrist-patient communication, more psychiatrist self-repair was associated with a better patient-rated therapeutic relationship (details available from the author on request). In non-medical interaction, self-repair has also been identified as an index of effort by conversational partners in sustaining mutual intelligibility in dialogue. ${ }^{15}$

Currently, mental health professionals receive little specific training, beyond basic communication and counselling skills, in how to communicate effectively with patients with psychosis. We developed a brief training programme for psychiatrists based on research findings that psychiatrist-patient shared understanding - assessed by means of psychiatrist 'self-repair' is associated with better relationships. ${ }^{14}$ The training was novel as it was developed from micro-analysis of psychiatrist-patient 
communication in previously recorded routine psychiatric encounters. It focused on the challenges of communicating in this therapeutic context along with effective ways of overcoming these challenges and empowering patients. This trial tested whether the novel training programme would increase psychiatrist self-repair and improve therapeutic relationships.

\section{Method}

\section{Study design}

This was a cluster randomised control trial (trial registration: ISRCTN94846422). Psychiatrists were randomised to the training or control group. Data were collected from psychiatrists and their patients at two time points: baseline (i.e. before psychiatrists received the training) and follow-up (i.e. at the first out-patient encounter after the training for each psychiatrist-patient pair (about 5 months after baseline)). At baseline and follow-up, encounters between participating psychiatrists and their patients were video-recorded in the clinic as usual. Researchers set up the camera and left the room. Psychiatrists and patients completed questionnaires.

\section{Sample size}

The target sample size was 72 patients (and 12 psychiatrists), i.e. 36 patients in each group, providing $85 \%$ power at the $5 \%$ significance level to detect an effect size of 1 (a doubling in the rate of repair) based on an assumed correlation between preand post-training measures of 0.7 and an intracluster correlation coefficient (ICC) of 0.65 in a previous study. ${ }^{16}$ As there was more psychiatrist turnover than anticipated during recruitment, additional participants were recruited so that the final sample size was 97 patients and 21 psychiatrists.

\section{Participants}

Recruitment took place between September 2011 and October 2012. Psychiatrist inclusion criteria were: specialist psychiatric trainees working in out-patient clinics or community mental health teams. Psychiatrists working at this level have basic knowledge and experience in psychiatry gained through at least 3 years' core psychiatric training and practice without direct supervision.

Patient inclusion criteria were: adults aged 18-65; meeting ICD-10 criteria for a diagnosis of schizophrenia or schizoaffective disorder $;{ }^{17}$ currently attending psychiatric out-patient clinics or being cared for by community mental health teams; and capable of giving informed consent. Exclusion criteria were: organic impairment or an encounter requiring an interpreter.

\section{Intervention}

The training was developed over 1 year by specialists in communication in psychiatry (R.M., D.K. and P.J.) and general medicine (A.C.) with input from patients. It was fully manualised (available in print/DVD on request or online, http://medicine.exeter. ac.uk/media/universityofexeter/medicalschool/profiles/TEMPO_ full_manual.pdf). Four sessions focused on: (a) understanding the patient with psychotic experiences: reflecting on the patient's experience and the professional and emotional response to psychotic symptoms; (2) communication techniques for working with positive and negative symptoms; (3) empowering the patient: agenda-setting at the start of the meeting and explaining/ normalising psychosis; and (4) involvement in decision-making about medication. ${ }^{2}$

The programme was based on previous research highlighting: engaging with the patient to acknowledge their distressing experience without an underlying goal of changing the patient's beliefs; ${ }^{10,18}$ negative symptoms as protective and working with patients with long-standing negative symptoms to set their own, albeit small, treatment goals; ${ }^{19}$ and involving the patient in decisions about treatment. ${ }^{2}$

The training was administered in four consecutive weekly group sessions lasting $3 \mathrm{~h}$ each and one individualised feedback session where participants reflected on their video-recorded communication with patients in the clinic. Each session was run by two facilitators (R.M., A.C., D.K. or P.B.). The weekly interval facilitated practising new skills with different patients and feeding back positive and negative experiences in the next session. The sessions were delivered to groups of up to nine participants. Each session included transcripts and video clips of each topic (e.g. delusions, agenda-setting, decision-making) in previously recorded psychiatrist-patient encounters using high levels of self-repair for each topic. ${ }^{1,2,10,18}$ Clips of excerpts, previously micro-analysed using conversation analysis, were played and then stopped to ask participants how they would respond to a particular patient utterance. This stimulated group discussions reflecting on alternative ways of communicating. This was followed by role-play, trying out new ways of communicating with each other and with simulated patients (professional actors) along with the use of real-time video feedback. The role-plays used actual scenarios from video-recorded encounters, for example an exchange where a patient wants to come off medication but the psychiatrist does not support this.

In the first session, psychiatrists participated in a simulated 'hearing voices exercise. ${ }^{20}$ Psychiatrists performed various tasks (e.g. a cognitive assessment) while listening to simulated voices. This exercise was highly rated by the participants, with most commenting on how distressing it was and that they now understood why patients feel a need to make sense of such experiences.

\section{Control condition}

Psychiatrists in the control condition did not receive the training and delivered treatment as usual.

\section{Framework for evaluating the training}

The framework for evaluating the training was that psychiatrists would feel more confident in communicating with patients with psychosis at the end of the training and apply the new communication skills - reflected behaviourally in increased use of self-repair - leading to improved therapeutic relationships from both psychiatrist and patient perspectives. Each of these outcomes was assessed.

\section{Outcome measures}

\section{Primary outcome}

Self-repair. The pre-determined primary outcome was psychiatrist self-repair in out-patient encounters with participating patients after the training, reflecting engagement with the patient and effort in establishing shared understanding. All pre- and post-training encounters were transcribed and selfrepair was automatically annotated on the transcripts using a computer program STIR (STrongly Incremental Repair) to detect self-repair. STIR detects the presence and extent of self-repairs, including repetitions, substitutions and deletions by detecting key phrases that indicate repair ('er', 'sorry', 'I mean', etc.), and using statistical measures of fluency and likely sentential sequence. The STIR program has been validated in clinical and non-clinical data and with people for whom English is not a first language. ${ }^{21,22}$ The accuracy (i.e. F-score) of the STIR algorithms' classifications 
of self-repairs applied to psychiatric data was $0.68 .^{21}$ To adjust for number of words spoken by each psychiatrist, self-repair was normalised by calculating mean number of self-repairs per 1000 words.

\section{Secondary outcomes}

Psychiatrist confidence. A self-rated questionnaire to assess psychiatrist self-confidence in communicating with patients with psychosis before and after the training was developed. Ten items (rated from 0 to 10 ) relating to each area in the training (e.g. 'I feel comfortable communicating with patients with negative symptoms', 'I feel comfortable explaining psychotic illness to patients'). A mean score was calculated, ranging from 0 to 10 , a higher score indicating higher self-confidence.

Therapeutic relationship. The therapeutic relationship was assessed using the Scale To Assess Therapeutic Relationship $(\mathrm{STAR})^{23}$ by each patient and psychiatrist at baseline and follow-up. The STAR scale was developed in a 4-year study beginning with item generation from interviews with patients and professionals and existing therapeutic relationship scales before rigorous psychometric validation. ${ }^{23}$ STAR has a patient and professional version. Each version has 12 items and 3 distinct factors: positive collaboration, positive clinician input and nonsupportive clinician input and emotional difficulties. The total score range is $0-48$ (a higher score equals a better relationship). Length of therapeutic relationship was documented.

Originally, a further follow-up point was planned, 6 months after the post-training encounters were recorded. However, this turned out to be impractical because many of the psychiatrists rotated posts after 1 year and so were no longer treating the patients in the trial.

\section{Procedure}

Consent was sought from individual psychiatrists prior to randomisation by the researchers on the study (P.J. and J.D.). Specialist psychiatric trainees working in out-patient clinics in East and North East London were identified. The number of eligible trainees was lower than anticipated. Hence, the inclusion criteria were widened to include fully qualified psychiatrists, i.e. staff and associate specialist grade (SASG) and consultant psychiatrists. Information letters were sent to 35 psychiatrists. Participating psychiatrists identified eligible patients in out-patient clinics. Eligible patients were approached by an independent researcher before their appointment with the psychiatrist, and were masked to whether their psychiatrist was part of the intervention or control group. Patients who provided written informed consent had their encounter video-recorded. When the training was complete, the next time each participating patient attended the clinic, this follow-up encounter was video-recorded. Ethical approval was granted by East London Research Ethics Committee 1 (10/H0703/12).

Psychiatrists' and patients' age, gender and ethnicity were recorded along with length of time psychiatrists had been qualified. Data were collected on patients' employment status and treatment history.

Researchers assessed symptoms on the 30-item Positive and Negative Syndrome Scale (PANSS) at baseline and follow-up. ${ }^{24}$ Researchers were trained in the assessment and interrater reliability was good $($ ICC $=0.90)$.

\section{Randomisation and masking}

Consenting psychiatrists were randomly allocated using simple randomisation in a 1:1 ratio to the control or intervention group. This was generated by the statistician (S.B.) using a sequence generated in Excel with the RAND function. There was no allocation concealment. Each psychiatrist was assigned to the next allocation in the sequence. The primary outcome, self-repair, was masked. For the secondary outcome, the therapeutic relationship, patients were masked but it was not possible to mask psychiatrists.

\section{Data analysis}

Data analysis was conducted in Stata 12.0. Data were summarised as numbers and percentages or means and standard deviations. Using all available cases, the adjusted treatment differences (intervention $v$. control group) along with $95 \%$ confidence intervals and $P$-values were estimated following intention-to-treat principles. The ICCs were estimated for each outcome using an adaptation of one-way analysis of variance which does not truncate negative ICCs at zero. ${ }^{25}$

For the primary outcome, self-repair, and for STAR psychiatrist, linear mixed effects regression models were fitted by restricted maximum likelihood, adjusting for baseline measure of the outcome and including a random effect (random intercept) for psychiatrist. Additionally, for STAR psychiatrist, patientreported number of months under the care of their psychiatrist was adjusted for.

For STAR patient, the estimated ICC was negative. Hence, a linear regression model ignoring clustering was fitted so as not to bias the standard error of treatment effect downwards. Baseline STAR patient score was adjusted for along with baseline PANSS total score based on previously reported negative associations between the PANSS and STAR patient. ${ }^{23}$

\section{Results}

\section{Psychiatrists}

Out of 35 psychiatrists that were approached, 25 (71\%) agreed to participate and were randomised. One psychiatrist was excluded before randomisation due to changing post. Four psychiatrists (control $n=2$, intervention $n=2$ ) had to be excluded after randomisation because they had too few eligible patients/changed post, leaving ten psychiatrists in the intervention group and eleven in the control group. All ten psychiatrists in the intervention group participated in training. Participant flow can be seen in the CONSORT diagram (Fig. 1). Psychiatrist characteristics are presented in Table 1 .

\section{Patients}

A total of 407 patients were eligible; $191 \mathrm{did}$ not attend their appointment. Twenty-five were not approached (considered too ill to approach for consent or appointment overlapped with another participant). Ninety-three did not consent. Of those approached, 97 patients (51\%) were recruited. An average of 4.6 patients (s.d. $=1.9$, range $1-7$ ) were recruited per psychiatrist: 5.0 patients $($ s.d. $=1.6)$ per psychiatrist in the intervention group and 4.3 patients $($ s.d. $=2.1$ ) per psychiatrist in the control group.

At baseline, patient data were collected from 97 patients: 96 encounters were video-recorded, 1 was missing due to equipment malfunction. Sixty-four patients were followed up and had the second encounter video-recorded. Patients could only be followed up if they were seeing the same psychiatrist again. Reasons for loss to follow-up are provided in Fig. 1.

There was an average of 152 days (approximately 5 months) between baseline and follow-up recordings (s.d. $=80.2$, range 47.2-500 days). The average baseline encounter length was $18.9 \mathrm{~min}$ (s.d. $=7.6$, range $7.3-37.1$ ) and at follow-up was $18.4 \mathrm{~min}$ (s.d. $=8.7$, range 4.0-43.5).

Patient characteristics are summarised in Table 2. 


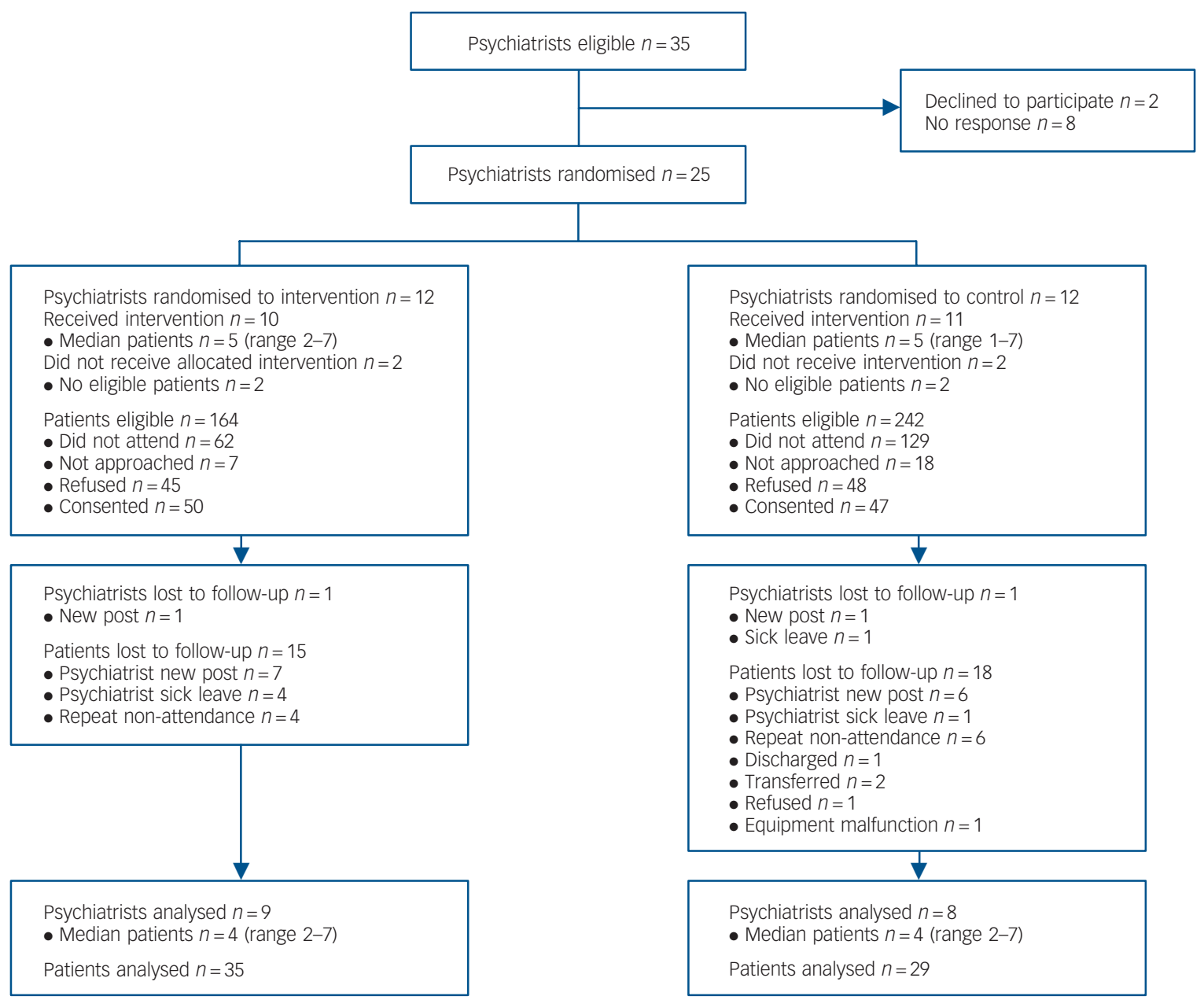

Fig. 1 CONSORT diagram showing participant flow in the study.

Associations between clinical characteristics and outcomes

At baseline, the Spearman correlation between patient-rated therapeutic relationship on the STAR and the patient-reported length of relationship with the psychiatrist was $r=-0.02$ $(P=0.85)$ The Spearman correlation between psychiatrist-rated therapeutic relationship on the STAR and the length of the relationship was $r=0.27(P=0.02)$.

\section{Primary outcome}

\section{Psychiatrist self-repair}

Psychiatrist self-repair was significantly higher in the intervention than the control group (Table 3; adjusted mean difference 6.4 selfrepairs per 1000 words, 95\% CI $1.46-11.33, P<0.011)$. The model-based ICC for self-repair was 0.03 : $3 \%$ of the variability in psychiatrist self-repair could be attributed to differences between psychiatrists. This corresponded to a large effect, Cohen's $d=0.91$.

\section{Secondary outcomes}

\section{Psychiatrist self-confidence}

Self-confidence in communicating with patients with psychosis increased. Paired $t$-tests showed a significant difference between scores before and after the training $(t=5.19,95 \%$ CI $1.0-2.4$, $P<0.01)$. The mean score increased by 1.7 points $(0-10$ scale $)$ from 6.9 (s.d. $=1.4)$ at baseline to $8.5($ s.d. $=1.2)$ at follow-up.
Therapeutic relationship

The quality of the therapeutic relationship improved significantly more in the intervention group than in the control group (Table 3 ), both on psychiatrist ratings (STAR mean difference 0.20, 95\% CI $0.03-0.37, P=0.022$ ) and patient ratings (STAR mean difference $0.21,95 \%$ CI $0.01-0.41, P=0.043)$. The ICC for the psychiatrist-rated STAR was 0.3 , i.e. $30 \%$ of the total variability in psychiatrists' ratings of the quality of the therapeutic relationship with their patients can be attributed to differences between psychiatrists. The ICC on the patient-rated STAR was negative, which, given the large sampling variation of ICCs, is most likely due to chance. The effect size for psychiatrist ratings of the relationship was $d=0.4$, a medium effect. The effect size for patient ratings of the relationship was $d=0.56$, a medium effect.

\section{Feedback on the training}

Attendance at the training was good (100\% participated in at least 3 of 4 sessions). Psychiatrists who could not attend a specific session received the session later or watched a video of the session. Psychiatrists rated the training as highly beneficial (mean score 8.9) on a $0-10$ scale (see Appendix for participant quotes).

\section{Change in communication}

Online Table DS1 shows examples of psychiatrist communication after the training for each of the four areas covered. For example, agenda-setting was one aspect of empowering the patient by 
Table 1 Psychiatrists' sociodemographic characteristics Intervention group Control group

\begin{tabular}{|lll|}
\hline$n$ & 10 & 11 \\
\hline Age, years: mean (s.d.) & $42.4(9.8)$ & $41.5(10.4)$ \\
\hline $\begin{array}{l}\text { Gender, } n \text { (\%) } \\
\text { Male }\end{array}$ & $8(80)$ & $7(64)$ \\
Female & $2(20)$ & $4(36)$ \\
\hline Grade, $n$ (\%) & & \\
Trainee (ST4-6) & $6(60)$ & $6(55)$ \\
SASG & $3(30)$ & $4(36)$ \\
Consultant & $1(10)$ & $1(9)$ \\
\hline Ethnicity, $n$ (\%) & $4(20)$ & $4(36)$ \\
White & $1(5)$ & $2(18)$ \\
Black & $4(20)$ & $5(45)$ \\
Asian & $1(10)$ & $0(0)$ \\
Mixed/other & & $6(55)$ \\
\hline First language, $n$ (\%) & $5(30)$ & $8.7(5.7)$ \\
English & $7(70)$ & \\
Other & $11.3(7.9)$ & \\
\hline Years in psychiatry, mean (s.d.) &
\end{tabular}

asking them what they wanted to talk about at the beginning of the meeting rather than 'Any questions?' when wrapping up. The following question was posed $40 \mathrm{~s}$ into the meeting: 'Well, the main thing would be perhaps today to understand what you would like from coming to meet with me today. What things did you want to talk about?'

\section{Discussion}

This study found that a brief intervention to enhance psychiatristpatient communication in the treatment of psychosis was effective. Psychiatrists' effort in establishing shared understanding with their patients was significantly higher after training. Both psychiatrists' and patients' views of the therapeutic relationship improved.

\section{Strengths and limitations}

The strengths of the study were that psychiatrist encounters in the clinic were video-recorded before and after training so that the change in communication could be compared between the control and the intervention group, adjusting for baseline communication. There was a range of experience among the psychiatrists and also varying lengths of relationships with their patients. The limitations were that the follow-up sample was smaller than at baseline because some psychiatrists changed post or were on sick leave. The participating patients may not be representative of all patients as they are likely to be more engaged in services and agreeable to participate in research. Moreover, the psychiatrists who participated may not be representative as they are likely to be more motivated than those who did not participate.

\section{Theoretical model: shared understanding}

The findings are in line with the theoretical model that guided the training and the trial. Psychiatrists' confidence in communicating with patients with psychosis improved. Communication was better after the training and the therapeutic relationship improved. This is the first communication intervention in mental healthcare to show these effects. Training in communication skills may benefit from an underlying theoretical model. The

\begin{tabular}{|c|c|c|}
\hline & Intervention group & Control group \\
\hline$n$ & 50 & 47 \\
\hline Age, years: mean (s.d.) & $43.8(10)$ & $42.8(10.4)$ \\
\hline \multicolumn{3}{|l|}{ Gender, $n(\%)$} \\
\hline Male & $32(64)$ & $34(72)$ \\
\hline Female & $18(36)$ & $13(28)$ \\
\hline \multicolumn{3}{|l|}{ Marital status, $n$ (\%) } \\
\hline Single & $36(72)$ & $36(76.5)$ \\
\hline Married/partnership & $10(20)$ & $7(15)$ \\
\hline Other & $4(8)$ & $4(8.5)$ \\
\hline \multicolumn{3}{|l|}{ Ethnicity, $n$ (\%) } \\
\hline White & $21(42)$ & $13(28)$ \\
\hline Black & $10(20)$ & $16(35)$ \\
\hline Asian & $12(24)$ & $11(24)$ \\
\hline Mixed/other & $7(14)$ & $6(13)$ \\
\hline \multicolumn{3}{|l|}{ First language, $n(\%)$} \\
\hline English & $36(74)$ & $37(80)$ \\
\hline Other & $13(26)$ & $9(20)$ \\
\hline \multicolumn{3}{|l|}{ Highest level of education, $n(\%)$} \\
\hline School & $24(51)$ & $19(40)$ \\
\hline Further education & $15(32)$ & $15(32)$ \\
\hline Higher education & $8(17)$ & $13(28)$ \\
\hline \multicolumn{3}{|l|}{ Employment status, $n$ (\%) } \\
\hline Unemployed & $30(61)$ & $32(70)$ \\
\hline Employed & $11(22)$ & 7 (15) \\
\hline Student/retired/other & $8(17)$ & $7(15)$ \\
\hline \multicolumn{3}{|l|}{$\begin{array}{l}\text { Number of hospital admissions, } \\
\text { mean (s.d.) }\end{array}$} \\
\hline Total previous & $3.3(4.2)$ & $3.6(7.5)$ \\
\hline Compulsory admissions & $1.2(1.4)$ & $2.0(2.4)$ \\
\hline \multicolumn{3}{|l|}{ Length of relationship with } \\
\hline psychiatrist, months: mean (s.d.) & $24.1(39.9)$ & $8.0(13.6)$ \\
\hline \multicolumn{3}{|l|}{ Symptoms (PANSS), mean (s.d.) } \\
\hline Total & $60.3(21.8)$ & $59.5(15.2)$ \\
\hline Positive & $15.5(7.1)$ & $14.9(6.8)$ \\
\hline Negative & $13.8(6.2)$ & $13.5(4.9)$ \\
\hline General & $31.0(10.8)$ & $31.0(8.5)$ \\
\hline
\end{tabular}

focus on self-repair may appear rather technical. However, with psychiatrists in the intervention group using $44 \%$ more self-repair than the control group, it appears to be a valid index of communicative interest in and engagement with the patient. The psychiatrists were not made aware that self-repair is considered to be positive or that self-repair would be assessed as an outcome. Hence, they are unlikely to have been consciously trying to do more of it in their communication. We would predict that trying to do more self-repair would, in itself, not be helpful. Rather, repairs are symptomatic of the effort a speaker is investing in producing an utterance that is tailored for their recipient. As such, they are likely to reflect a shift in thinking about the role of communication and genuine adjustments to find the best possible expression.

\section{Taking the listener's needs into account: preventing misunderstanding and displaying sensitivity}

To illustrate the kinds of self-repairs used in practice and the different ways in which they can take a listener's needs into account, some examples are provided. In the following utterance, the psychiatrist states 'I mean it would be good if you can keep up 


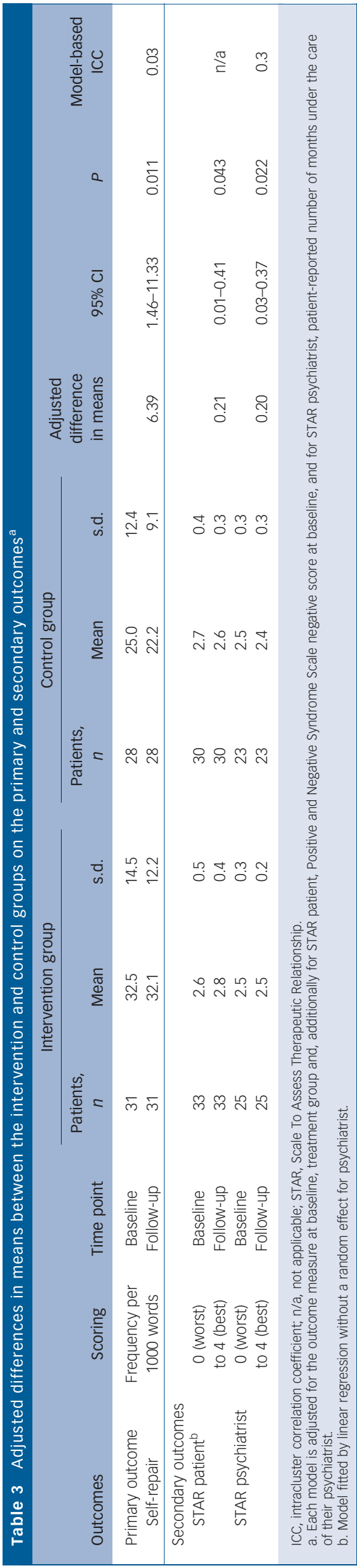

with the healthy life style, I mean er, not to take the medication if not really necessary'. Here 'healthy life style' is qualified (repaired) to 'not to take the medication' as a number of lifestyle factors have previously been discussed including diet and exercise. In this example, the self-repair clarifies a referent and prevents a possible misunderstanding by the patient. In the next example, the psychiatrist has proposed that it may be worth exploring how the patient could gain some more control over their overpowering voices rather than taking such a high dose of medication. The patient is somewhat resistant to the term 'control'. In line 5, the psychiatrist amends 'control' in a sensitive adjustment to the patient's perspective.

1. Patient: But isn't controlling wrong in a sense? Strange.

2. Doctor:

It it.

3. Doctor: It it it it.

4. Patient: You can actually control er.

5. Doctor: Er, when I say control, IIII'm, I think more in living, in terms of living with them, yeah?

Finally, in the excerpt below, the psychiatrist and patient have been discussing the patient's mother's recent death and the general question format 'How have things been in the past few months?' is revised quickly to 'I mean, I know that your day kind of revolved around [your mother]', displaying sensitivity to the patient's particular circumstances. Not revising the question from its first version would be hearable as insensitive to how the patient's life has been affected by their mother's death. Revising it in this way indicates a sensitive affective stance towards the patient.

Doctor: So how have things been in the past few months, I mean, I know that your day kind of revolved around your mother?

Patient: My day revolves around seeing my brother and sister a lot now, now my Mum's no longer with us.

It is interesting that psychiatrist communicative engagement decreased in the control group but was maintained in the intervention group. This is consistent with psychiatrists' reports in the training on the challenges of communicating about psychotic experiences over time, i.e. when they first meet patients, they are more engaged in their experiences but this can be challenging to maintain when patients are keen to talk about experiences repeatedly. A focus on self-repair, as an index of engagement with patients, may be useful in training and in research. Psychiatrists identified the training as filling a gap in their training. In research, the current theoretical model could be applied in other disorders and treatment settings to advance the field of communication skills in psychiatry and medicine.

\section{Improving the therapeutic relationship}

Psychiatrists' ratings of the therapeutic relationship improved considerably (a medium effect size). Although the confidence intervals are reasonably wide, possibly due to the sample size, this is the first study to show an improvement of the therapeutic relationship through training and suggests that the proposed mechanism of effect (i.e. increased communicative engagement with the patient) does indeed improve the quality of the therapeutic relationship. This finding is encouraging given that, in psychiatric treatment, the professional's rating of the relationship appears to be a stronger predictor of outcome than the patient's rating, ${ }^{26}$ the reverse of psychotherapy. Given that the odds of having adherent patients are twice as high if there is a good doctorpatient relationship, ${ }^{5}$ this is an important locus of intervention in improving longer-term outcomes. The current study was designed to investigate the effect of the intervention on process outcomes. Future studies would be required to investigate 
longer-term outcomes such as symptoms, quality of life and social functioning.

There is increasing interest in harnessing the potential of the therapeutic relationship in psychiatry. ${ }^{27}$ Two interventions have focused on helping patients to prioritise what they wish to discuss with their clinician. Priebe $e t a l^{28}$ found that a computer-mediated intervention to structure care coordinator-patient communication improved quality of life, reduced unmet needs for care and improved treatment satisfaction. Van Os et $a l^{29}$ used a checklist which patients completed before seeing their psychiatrist, which improved patient-reported quality of the communication and increased changes in management. Meanwhile, there is considerably more research in other fields such as primary care and oncology. ${ }^{30}$ As Fallowfield et al have noted, senior oncology doctors acknowledge that lack of communication training in complex medical contexts contributes to psychological morbidity, emotional burnout and depersonalisation. ${ }^{31}$ Psychiatrists are also at risk of these negative outcomes. Many psychiatrists are highly skilled communicators addressing complex problems, and some of the training was based on identifiying what they are doing in everyday practice. Specifying these skills and integrating them in psychiatric training would address the need within the profession to define the skills that psychiatrists use in treating complex mental health problems. ${ }^{8,32,33}$

This is the first study to test an intervention for psychiatrists to enhance communication with patients with psychosis. It suggests that shared understanding, which can be challenging in the treatment of psychosis, can be targeted in training and is important for improving the quality of communication and the therapeutic relationship.

Rose McCabe, PhD, University of Exeter Medical School, Exeter; Paula John, Dipl-Psych, Newham Centre for Mental Health, Barts and The London School of Medicine and Dentistry, Queen Mary University of London, London; Jemima Dooley BA, University of Exeter Medical School, Exeter; Patrick Healey, PhD, Cognitive Science Research Group, School of Electronic Engineering and Computer Science, Queen Mary University of London; Annie Cushing, PhD, Centre for Medical Education, Institute of Health Sciences Education, Barts and The London School of Medicine and Dentistry, Queen Mary University of London, St Bartholomew's Hospital, London; David Kingdon, MD, Department of Psychiatry, University of Southampton, Southampton; Stephen Bremner, PhD, Stefan Priebe, FRCPsych, Newham Centre for Mental Health, Barts and The London School of Medicine and Dentistry, Queen Mary University of London, London, UK

Correspondence: Professor Rose McCabe, Room 1.05, College House, University of Exeter, St Luke's Campus, Heavitree Road, Exeter EX1 2LU, UK. Email: r.mccabe@exeter.ac.uk

First received 7 Dec 2015, accepted 12 Dec 2015

\section{Funding}

The TEMPO study was funded by the National Institute for Health Research's (NIHR) Research for Patient Benefit Programme (PB-PG-0408-16279). The work was also supported by the NIHR Collaboration for Leadership in Applied Health Research and Care South West Peninsula at the Royal Devon and Exeter NHS Foundation Trust. The funder had no role in study design, data collection, data analysis, interpretation, writing of the report, or decision to submit for publication. All study researchers were independent from the funder. The views are those of the author(s) and not necessarily those of the NHS, the NIHR or the Department of Health.

\section{Acknowledgements}

We are most grateful to the participating psychiatrists and patients, Dr Peter Byrne for delivering training, Husnara Khanom and Mary Lavelle for assistance with data collection, and Chris Howes and Julian Hough for assistance with self-repair annotation. We also thank the anonymous reviewers for their constructive comments in revising the manuscript.

\section{Appendix}

\section{Participant perspectives on the training}

'The hearing voices exercise was very powerful. I now understand why patients want to talk about their voices.'
'How to explore in depth patients' concerns and listen actively with more attention to patients' cues.'

'EAR (Explore, Active Listening and Respond) and GUNS (Give overview of options, Understanding check, Negotiate, Summarise decision) were excellent!'

'I learned useful approaches and insight into my abilities (both strength and weaknesses) as a psychiatrist'

'Understanding that I need to explore patients' concerns before coming to a decision-making stage.'

'The art of discussion and negotiation in sharing decisions.'

'Paying more attention to the patient's agenda and their priorities

reduces the feeling of yet another routine.'

'Goal-setting with patients with negative symptoms - realistic and achievable.'

'Thinking about the patient perspective of psychosis.'

'Advanced techniques for explaining psychotic symptoms.'

'Even more of a focus on conflict and disagreement could be good.'

'I thought I knew a lot about the story of his delusions, but going through

the "voices checklist" makes me realise that I don't really how and when it started and so on.'

'I've never realised how much taking notes in the consultation affects the connection with the patient.

'It sounds as if I'm just going through a checklist, rather than talking to the patient."

'I should invite the patient to ask more questions.'

\section{References}

1 Priebe S, Dimic S, Wildgrube C, Jankovic J, Cushing A, McCabe R. Good communication in psychiatry - a conceptual review. Eur Psychiatry 2011; 26 403-7.

2 McCabe R, Khanom H, Bailey $\mathrm{P}$, Priebe $\mathrm{S}$. Shared decision-making in ongoing outpatient psychiatric treatment. Patient Educ Couns 2013; 91: 326-28.

3 Day J, Bentall R, Roberts C, Randall F, Rogers A, Cattell D. Attitudes toward antipsychotic medication: the impact of clinical variables and relationships with health professionals. Arch Gen Psychiatry 2005; 62: 717-25.

4 Priebe S, Richardson M, Cooney M, Adedeji O, McCabe R. Does the therapeutic relationship predict outcomes of psychiatric treatment in patients with psychosis? A systematic review. Psychother Psychosom 2011; 80: 70-7.

5 Zolnierek KBH, Matteo D, Robin M. Physician communication and patient adherence to treatment: a meta-analysis. Med Care 2009; 47: 826-34.

6 Johansson $\mathrm{H}$, Eklund M. Patients' opinion on what constitutes good psychiatric care. Scand J Caring Sci 2003; 17: 339-46.

7 Bhugra D, Sivakumar K, Holsgrove G, Butler G, Leese M. What makes a good psychiatrist? A survey of clinical tutors responsible for psychiatric training in the UK and Eire. World Psychiatry 2009; 8: 119-20.

8 Drew P, Chatwin J, Collins S. Conversation analysis: a method for research into interactions between patients and health-care professionals. Health Expect 2001; 4: 58-70.

9 Heritage J, Robinson JD, Elliott MN, Beckett M, Wilkes M. Reducing patients' unmet concerns in primary care: the difference one word can make. J Gen Intern Med 2007; 22: 1429-33.

10 McCabe R, Heath C, Burns T, Priebe S. Engagement of patients with psychosis in the consultation: conversation analytic study. BMJ 2002; 325: 1148-51.

11 Schegloff $E$, Jefferson $G$, Sacks $H$. The preference for self-correction in the organisation of repair in conversation. Language 1977; 53: 361-82.

12 Hayashi M, Raymond G, Sidnell J. Conversational Repair and Human Understanding. Cambridge University Press, 2013.

13 Brennan S, Schober M. How listeners compensate for disfluencies in spontaneous speech. J Mem Lang 2001; 44: 274-96.

14 Themistocleous M, McCabe R, Rees N, Hassan I, Healey P, Priebe S. Establishing mutual understanding in interaction: an analysis of conversational repair in psychiatric consultations. Commun Med 2009; 6 : 165-76.

15 Colman M, Healey PGT. The distribution of repair in dialogue. In Proceedings of the 33rd Annual Meeting of the Cognitive Science Society (eds L Carlson, TF Shipley): 1563-8. Boston, Massachusetts, 20-23 July 2011.

16 McCabe R, Healey PGT, Priebe S, Lavelle M, Dodwell D, Laugharne R, et al. Shared understanding in psychiatrist-patient communication: association with treatment adherence in schizophrenia. Patient Educ Couns 2013; 93: 73-9.

17 World Health Organization. The ICD-10 Classification of Mental and Behavioural Disorders: Clinical Descriptions and Diagnostic Guidelines. WHO, 1992 
18 Federico M, Priebe S, Fusco C, Strapelli N, Singh R, McCabe R. Communication about psychotic symptoms in long-term psychiatric care Psychopathology 2012; 46: 233-40.

19 Kingdon DJ, Turkington D. Cognitive Therapy of Schizophrenia. Guilford Press, 2008.

20 Deegan PA. Hearing Voices That Are Distressing: A Training and Simulated Experience. Lawrence, 1996.

21 Howes C, Hough J, Purver M, McCabe R. Helping, I mean assessing psychiatric communication: an application of incremental self-repair detection. In Proceedings of the 18th SemDial Workshop on the Semantics and Pragmatics of Dialogue (Dia/Watt): 80-9. Edinburgh, 1-3 September 2014 (http://www.macs.hw.ac.uk/InteractionLab/Semdial/semdial14.pdf).

22 Hough J, Purver M. Strongly incremental repair detection. In Proceedings of the 2014 Conference on Empirical Methods in Natural Language Processing (EMNLP): 78-89. Doha, Qatar; 2014 (http://emnlp2014.org/papers/pdf/ EMNLP2014009.pdf).

23 McGuire-Snieckus R, McCabe R, Catty J, Hansson L, Priebe S. A new scale to assess the therapeutic relationship in community mental health care: STAR. Psychol Med 2007; 37: 85-95.

24 Kay S, Fiszbein A, Opler L. The positive and negative syndrome scale (PANSS) for schizophrenia. Schizophr Bull 1987; 13: 261-76.

25 Ukoumunne OC. A comparison of confidence interval methods for the intraclass correlation coefficient in cluster randomized trials. Stat Med 2002 21: $3757-74$.
26 McCabe R, Bullenkamp J, Hansson L, Lauber C, Martinez-Leal R, Rössler W, et al. The therapeutic relationship and adherence to antipsychotic medication in schizophrenia. PLOS ONE 2012; 7: e36080.

27 Arnow BA, Steidtmann D. Harnessing the potential of the therapeutic alliance. World Psychiatry 2014; 13: 238-40.

28 Priebe S, McCabe R, Bullenkamp J, Hansson L, Lauber C, Martinez-Leal R, et al. Structured patient-clinician communication and 1-year outcome in community mental healthcare: cluster randomised controlled trial. Br J Psychiatry 2007; 191: 420-6.

29 Van Os J, Altamura AC, Bobes J, Gerlach J, Hellewell JS, Kasper S, et al. Evaluation of the Two-Way Communication Checklist as a clinical intervention. Results of a multinational, randomised controlled trial. Br J Psychiatry 2003; 184: 79-83.

30 Dwamena F, Holmes-Rovner M, Gaulden C, Jorgenson S, Sadigh G, Sikorskii A et al. Interventions for providers to promote a patient-centred approach in clinical consultations (Review). Cochrane Database Syst Rev 2012; 12: CD003267.

31 Fallowfield L. Can we improve the professional and personal fulfilment of doctors in cancer medicine? Br J Cancer 1995; 71: 1131-3.

32 Bhugra D. Alienated alienists: a new hope? Lancet Psychiatry 2014; 1: 257-9.

33 Maj M. Technical and non-technical aspects of psychiatric care: the need for a balanced view. World Psychiatry 2014; 13: 209-10.

\section{psychiatry in history}

\section{'The Maudsley at War' exhibition at the RCPsych London}

Itoro Udo

War has contributed immensely to our discovery, understanding and advancing of treatments for post-traumatic stress disorder (PTSD). This exhibition aimed to showcase the influence of the then Maudsley Neurological Clearing Hospital in relieving the mental suffering of soldiers during the First World War.

Sir Frederick Mott (1853-1926) was a war-time psychiatrist who is less well known than Henry Maudsley (1835-1918), but from this exhibition it is clear that he worked tirelessly to manage the different presentations of what was then referred to as shel shock. He believed in diversion of the mind as a treatment principle. Injured soldiers were encouraged to focus on therapeutic activities and away from their experiences of war in order to promote healing. Mott also believed in the vital contribution of a structured, managed external environment to aid the healing

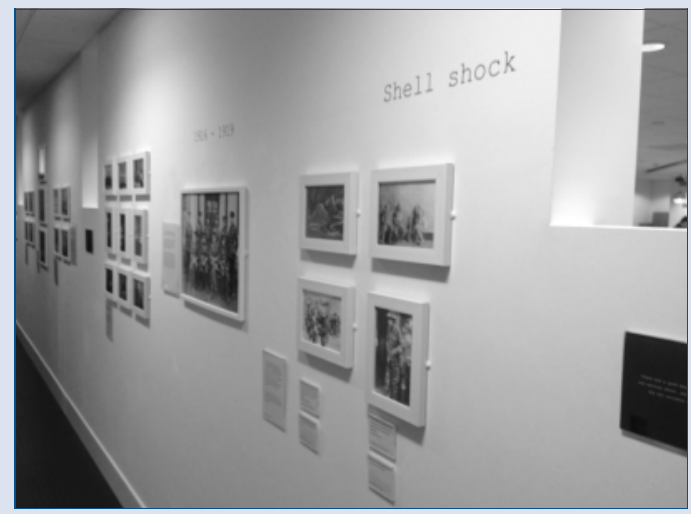

A photograph of the exhibition at the college taken by the author. process. This involved recreation activities and spaces, colours of bedrooms, nutritious food and rest so as to promote an 'atmosphere of cure'. The hospital had a gymnasium where soldiers used parallel bars, climbing and skipping ropes, etc. Sir Frederick persuaded benefactors to support occupational workspaces where soldiers took part in activities including carpentry, gardening and book-binding.

This Hospital had enormous reach. The British Army reported over 80000 cases of shell shock during the First World War and at least 12400 are thought to have been treated there. Initially, it had 144 beds but this expanded to 450 by 1917 . The soldiers experienced symptoms such as sleep paralysis, nightmares, palpitations, fatigue, functional paralysis. The official position was to restore them to health and send them back to the war front but we are informed by the exhibition that only about $25 \%$ returned.

Mott's use of diversion of the mind is reminiscent of current treatment modalities of creating or managing dissociation in the management of severe manifestations of PTSD, conversion and somatoform disorders. His ideas of an 'atmosphere of cure' is mirrored in treatments today. We continue to use structured living spaces in intellectual disability and forensic psychiatry. It is also what we try to create in psychodynamic therapy. There is also increasing use of nidotherapy in the management of severe mental disorders. It is interesting that even at that time, it was believed (Howard Kemp-Prosser) that bedrooms painted in certain colours were more restorative to the mind. The treatments were holistic, encompassing diet and work. We are becoming curious again about how diet may aid recovery from mental illness. What was missing from this exhibition are details of therapies or medications used in managing these soldiers. Pictures of equipment would have been an interesting addition.

These sorts of illnesses and symptoms still continue to plague our society. We know more about them today but provision of services, access to treatments and funding for research continue to be insufficient. Patients who experience today what we may call medically unexplained symptoms often suffer from lack of clear and coherent treatment pathways. Government investments have not addressed these as seriously as policies had intended. The exhibition illustrates that management of these disorders was and still is resource intensive. Then and now, it requires multidisciplinary, collaborative and stepped care. 